\title{
Theory of the density fluctuation spectrum of strongly correlated electrons
}

\author{
G. Khaliullin and P. Horsch \\ Max-Planck-Institut für Festkörperforschung, Heisenbergstr. 1, D-70569 Stuttgart (Germany)
}

(May 14, 1996)

\begin{abstract}
The density response function $N(\mathbf{q}, \omega)$ of the twodimensional $t-J$ model is studied starting from a mixed gauge formulation of the slave boson approach. Our results for $N(\mathbf{q}, \omega)$ are in remarkable agreement with exact diagonalization studies, and provide a natural explanation of the anomalous features in the density response in terms of the spin polaron nature of the charge carriers. In particular we have identified unexplained low energy structures in the diagonalization data as arising from the coherent polaron motion of holes in a spin liquid.

PACS numbers: 71.27.+a, 71.45.Gm, 74.20.Mn
\end{abstract}

Recent exact diagonalization studies [1,2] of the dynamical density response $N(\mathbf{q}, \omega)$ at large momentum transfer have revealed several features unexpected from the point of view of weakly correlated fermion systems: (i) a strong suppression of low energy $2 k_{F}$ scattering in the density response, (ii) a broad incoherent peak whose shape is rather insensitve to hole concentration and exchange interaction $J$, (iii) the very different form of $N(\mathbf{q}, \omega)$ compared to the spin response function $S(\mathbf{q}, \omega)$, which share common features in usual fermionic systems.

While considerable analytical work has been done to explain the spin response of the $t-J$ model [3] only few authors analysed $N(\mathbf{q}, \omega)$. Wang et al. [4] studied collective excitations in the density channel and found sharp peaks at large momenta corresponding to free bosons. Similar results were obtained by Gehlhoff and Zeyher [5] using the X-operator formalism. Lee et al [6] considered a model of bosons in a fluctuating gauge field and found a broad incoherent density fluctuation spectrum at finite temperature, due to the coupling of bosons to a quasistatic disordered gauge field.

The aim of our paper is to show that the essential features observed in the numerical studies can be obtained in the framework of the Fermi-liquid phase of the $t-J$ model at zero temperature. Our main findings are: (i) at low momenta the main effect of strong correlations is to transfer spectral weight from particle-hole excitations into a pronounced collective mode. Because of the strong damping of this mode (linear in $q$ ) due to the coupling to the spinon particle-hole continuum, this collective excitation is qualitatively different from a sound mode. (ii) At large momenta we find a strict similarity of $N(\mathbf{q}, \omega)$ with the spectral function of a single hole moving in a uniform RVB spinon background. In this regime $N(\mathbf{q}, \omega)$ consists of a broad peak at high energy whose origin is the fast, incoherent motion of bare holes. The polaronic nature of dressed holes leads to the formation of a second peak at lower energy, which is more pronounced in $(\pi, 0)$ direction in agreement with diagonalization studies [1, 2]. For the static structure factor we find good agreement with numerical results [7], 8 ].

Following Kotliar and Liu [9] and Wang et al [4] we start from the $\mathrm{N}$-component generalization of the slaveboson $t$ - $J$ Hamiltonian, $H_{t J}=H_{t}+H_{J}$ :

$$
\begin{aligned}
& H_{t}=-\frac{2 t}{N} \sum_{<i, j>\sigma}\left(f_{i \sigma}^{+} h_{j}^{+} h_{i} f_{j \sigma}+\text { h.c. }\right), \\
& H_{J}=\frac{J}{N} \sum_{<i, j>\sigma \sigma^{\prime}} f_{i \sigma}^{+} f_{i \sigma^{\prime}} f_{j \sigma^{\prime}}^{+} f_{j \sigma},
\end{aligned}
$$

where $\sigma=1, \cdots, N$ is the fermionic channel index, and $h_{i}$ denotes the bosonic holes. The number of auxiliary particles must obey the constraint $\sum_{\sigma} f_{i \sigma}^{+} f_{i \sigma}+h_{i}^{+} h_{i}=$ $N / 2$. The original $t-J$ model is recovered for $N=2$.

The slave boson parametrization provides a straightforward description of the strong suppression of density fluctuations of constrained electrons through the representation of the density response in terms of a dilute gas of bosons. A common treatment of model (1) is the density-phase representation ("radial" gauge [10]) of the bosonic operator $h_{i}=r_{i} \exp \left(i \theta_{i}\right)$ with the subsequent $1 / N$-expansion around the Fermi-liquid saddle point. While this gauge is particularly useful to study the low energy and momentum properties, it is not very convenient for the study of the density response in the full $\omega$ and $\mathbf{q}$ space. Formally the latter follows in the radial gauge from the fluctuations of $r_{i}^{2}$. If one considers for example convolution type bubble diagrams, one realizes that their contribution to the static structure factor is correctly of order $1 / N$, but is not proportional to the density of holes $\delta$ as it should be. According to Arrigoni et al [11] such unphysical results originate from a large negative pole contribution in the $\left\langle r_{-\mathbf{q}} r_{\mathbf{q}}\right\rangle_{\omega}$ Green's function of the real field $r$, which is hard to control by a perturbative treatment of phase fluctuations. We follow therefore Popov 12 using the density-phase treatment only for small momenta $q<q_{0}$, while keeping the original particle-hole representation of the density operator, $b^{+} b$, at large momenta. More precisely $h_{i}=r_{i} \exp \left(i \theta_{i}\right)+b_{i}$, where $b_{i}=\sum_{|\mathbf{q}|>q_{0}} h_{\mathbf{q}} \exp \left(i \mathbf{q} \mathbf{R}_{i}\right)$. The cutoff $q_{0}$ is introduced dividing "slow" (collective) variables represented by $r$ and $\theta$ from "fast" (single-particle) degrees of freedom. As explained by Popov 12] this "mixed" gauge 
is particularly useful for finite temperature studies to control infrared divergences. We start formally with "mixed" gauge and keep only terms of order $\delta$ and $1 / N$ in the bosonic self energies. In this approximation our zero temperature calculations become quite straightforward: The cutoff $q_{0}<\delta$ actually does not enter in the results and we arrive finally at the Bogoliubov theory for a dilute gas of bosons moving in a fluctuating spinon background.

The Lagrangian corresponding to the model (1) is then given by (the summation over $\sigma$ is implied)

$$
\begin{aligned}
L & =\sum_{i}\left(f_{i \sigma}^{+}\left(\frac{\partial}{\partial \tau}-\mu_{f}\right) f_{i \sigma}+b_{i}^{+}\left(\frac{\partial}{\partial \tau}-\mu_{b}\right) b_{i}\right)+H_{t}+H_{J} \\
& +\frac{i}{\sqrt{N}} \sum_{i} \lambda_{i}\left(f_{i \sigma}^{+} f_{i \sigma}+\left(r_{i}+b_{i}^{+}\right)\left(r_{i}+b_{i}\right)-\frac{N}{2}\right),
\end{aligned}
$$$$
H_{t}=-\frac{2 t}{N} \sum_{<i j>} f_{i \sigma}^{+} f_{j \sigma}\left(b_{j}^{+} b_{i}+r_{i} r_{j}+r_{j} b_{i}+b_{j}^{+} r_{i}\right)+\text { h.c. }
$$

Here the $\lambda$ field is introduced to enforce the constraint, and $\mu_{f}, \mu_{b}$ are fixed by the particle number equations $\left\langle n_{f}\right\rangle=\frac{N}{2}(1-\delta)$ and $\left\langle r_{i}^{2}+b_{i}^{+} b_{i}\right\rangle=\frac{N}{2} \delta$, respectively. The uniform mean field solution $r_{i}=r_{0} \sqrt{N / 2}$ leads in the large $N$ limit to the renormalized narrow fermionic spectrum $\xi_{\mathbf{k}}=-z \tilde{t} \gamma_{\mathbf{k}}-\mu_{f}$, with $\tilde{t}=J \chi+t \delta, \gamma_{\mathbf{k}}=$ $\frac{1}{2}\left(\cos k_{x}+\cos k_{y}\right), \chi=\sum_{\sigma}\left\langle f_{i \sigma}^{+} f_{j \sigma}\right\rangle / N$, and $z=4$ the number of nearest neighbors. Distinct from the finitetemperature gauge-field theory of Nagaosa and Lee 13 the bond-order phase fluctuations acquire a characteristic energy scale in this approach [4], and the fermionic ("spinon") excitations can be identified with Fermi-liquid quasiparticles. The mean field spectrum of bosons is $\omega_{\mathbf{q}}=2 z \chi t\left(1-\gamma_{\mathbf{q}}\right)$. Thus the effective mass of holes $m_{h}^{0} \propto 1 / t$ is much smaller than that of the spinons.

Due to the diluteness of the bosonic subsystem, $\delta \ll 1$, the density correlation function is mainly given by the condensate induced part which is represented by the Green's function $\left\langle\left(b_{\mathbf{q}}^{+}+b_{-\mathbf{q}}\right)\left(b_{\mathbf{q}}+b_{-\mathbf{q}}^{+}\right)\right\rangle_{\omega}$ for $q>q_{0}$, and $2\left\langle r_{-\mathbf{q}} r_{\mathbf{q}}\right\rangle_{\omega}$ for $q<q_{0}$, respectively. The $1 / N$ self-energy corrections to these functions are calculated in a conventional way [10,9] expanding $r_{i}=\left(r_{0} \sqrt{N}+(\delta r)_{i}\right) / \sqrt{2}$ and considering Gaussian fluctuations around the mean field solution. Neglecting all terms of order $\delta / N$ and $q_{0}^{2} / N$, only one relevant $1 / N$ contribution remains which corresponds to the dressing of the slave-boson Green's function by spinon particle-hole excitations. Within this approximation and at zero temperature no divergences occur at low momenta, thus one can take the limit $q_{0} \rightarrow 0$. The final result for the dynamic stucture factor (normalized by the hole density) is:

$$
\begin{aligned}
& N_{\mathbf{q}, \omega}=\frac{2}{\pi} \operatorname{Im}\left(\left(\omega_{\mathbf{q}} a+S_{\mathbf{q}, \omega}^{(1 / N)}-\mu_{b}\right) / D_{\mathbf{q}, \omega}\right), \\
& D_{\mathbf{q}, \omega}=\left(\omega_{\mathbf{q}} a+S_{\mathbf{q}, \omega}^{(1 / N)}-\mu_{b}\right)\left(\omega_{\mathbf{q}}+S_{\mathbf{q}, \omega}^{(1)}+S_{\mathbf{q}, \omega}^{(1 / N)}-\mu_{b}\right)
\end{aligned}
$$

$$
-\left(\omega a-A_{\mathbf{q}, \omega}^{(1 / N)}\right)^{2} .
$$

The origin of the contribution

$$
\begin{aligned}
S_{\mathbf{q}, \omega}^{(1)} & =z \operatorname{tr}_{0}^{2}\left(\frac{\left(1+\Pi_{2}\right)^{2}}{\Pi_{1}}-\Pi_{3}\right)_{\mathbf{q}, \omega} \\
\Pi_{m} & =z t \sum_{\mathbf{k}} \frac{n\left(\xi_{\mathbf{k}}\right)-n\left(\xi_{\mathbf{k}+\mathbf{q}}\right)}{\xi_{\mathbf{k}+\mathbf{q}}-\xi_{\mathbf{k}}-\omega-i 0^{+}}\left(\gamma_{\mathbf{k}}+\gamma_{\mathbf{k}+\mathbf{q}}\right)^{m-1},
\end{aligned}
$$

is the indirect interaction of bosons via the spinon band due to the hopping term (which gives $\Pi_{3}$ in (4)) and due to the coupling to spinons via the constraint field $\lambda$. The latter channel provides a repulsion between bosons, making $S^{(1)}(\omega=0)$ positive and therefore ensuring the stability of the uniform mean-field solution. The $1 / N$ self energies $S^{(1 / N)}$ and $A^{(1 / N)}$ are essentially a single boson property. They are given by the symmetric and antisymmetric combinations (with respect to $\omega+i 0^{+} \rightarrow$ $\left.-\omega-i 0^{+}\right)$of the self energy

$\Sigma_{\mathbf{q}, \omega}^{(1 / N)}=\frac{4}{N} \sum_{|\mathbf{k}|<k_{F}<\left|\mathbf{k}^{\prime}\right|}\left(z t \gamma_{\mathbf{k}^{\prime}-\mathbf{q}}\right)^{2} G_{\mathbf{q}+\mathbf{k}-\mathbf{k}^{\prime}}^{0}\left(\omega+\xi_{\mathbf{k}}-\xi_{\mathbf{k}^{\prime}}\right)$.

Here $G_{\mathbf{q}}^{0}(\omega)=\left(\omega-\omega_{\mathbf{q}}-\Sigma_{\mathbf{q}, \omega}^{(1 / N)}+\mu_{b}\right)^{-1}$ is the Green's function for a single slave boson moving in a uniform RVB background. Although in the context of $1 / N$ theory the $G^{0}$ function in (5) should be considered as a free propagator, we shall use here the selfconsistent polaron picture for a single hole [14]. This is important when comparing the theory for $N=2$ with diagonalization studies. Finally, the constants $a$ and $\mu_{b}$ in (3) are given by $\left(1-t r_{0}^{2} / \tilde{t}\right)$ and $S^{(1 / N)}(\omega=\mathbf{q}=0)$, respectively.

In the small $\omega, \mathbf{q}$ limit $N(\mathbf{q}, \omega)$ (3) is mainly controlled by the interaction of bosons represented by the $S^{(1)}$ term, while the internal polaron structure of the boson determined by $S^{(1 / N)}$ is less important even for $N=2$, as expected on physical grounds. In this limit our results are

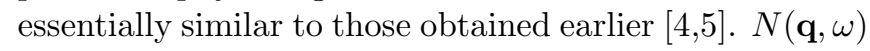
consists of a weak spinon particle-hole continuum with cutoff $\propto v_{F} q$, and a very pronounced collective mode which nearly exhausts the sum rule. We find that the velocity of this mode is always somewhat smaller than the Fermi velocity, $v_{s} \leq v_{F} \simeq z \tilde{t}$, and therefore in a strict sense there is no well defined sound. Since the spinonboson coupling does not vanish in the limit $q \rightarrow 0$ the imaginary part of the collective excitation pole is linear in $\omega$ (or $q$ ), thus the damping is only numerically small compared with the excitation energy.

The density response $N(\mathbf{q}, \omega)$ at large momenta, $q>\delta$, which we can compare with diagonalization results, is dominated by the properties of a single boson dressed by spinon excitations. For the numerical calculation of $N(\mathbf{q}, \omega)$ given by Eqs. (3-5) we evaluate in a first step the single hole self-energy $\Sigma_{\mathbf{q}, \omega}^{(1 / N)}$ in a selfconsistent way. 


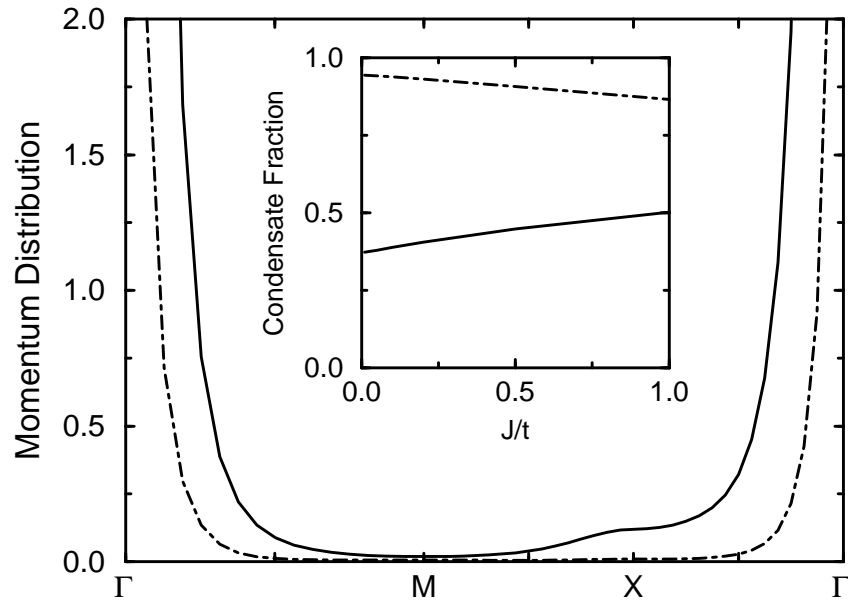

FIG. 1. Momentum distribution $\tilde{n}_{\mathbf{q}}$ normalized by $\delta$, for $\delta=0.25$ and $J / t=0.4$. Inset: Condensate fraction $r_{0}^{2} / \delta$ versus $\mathrm{J}$ for $\delta=0.25$. Comparison of calculation with (solid line) and without (dash-dotted) inclusion of polaron effects.

Then the parameter $r_{0}^{2}$ in (3-4), which formally corresponds to the condensate fraction in our theory, is found selfconsistently from $r_{0}^{2}=\delta-\sum_{\mathbf{q} \neq 0} \tilde{n}_{\mathbf{q}}$.

The momentum distribution $\tilde{n}_{\mathbf{q}}=\left\langle b_{\mathbf{q}}^{+} b_{\mathbf{q}}\right\rangle$ is calculated using the corresponding bosonic Green's function for finite hole-density:

$$
\begin{aligned}
G_{\mathbf{q}, \omega}=-( & \omega a-A_{\mathbf{q}, \omega}^{(1 / N)}+\omega_{\mathbf{q}}\left(1-\frac{t r_{0}^{2}}{2 \tilde{t}}\right) \\
& \left.+\frac{1}{2} S_{\mathbf{q}, \omega}^{(1)}+S_{\mathbf{q}, \omega}^{(1 / N)}-\mu_{b}\right) / D_{\mathbf{q}, \omega} .
\end{aligned}
$$

While the $1 / q$ behavior of $\tilde{n}_{\mathbf{q}}$ (Fig.1) at small momenta, $q \leq \delta$, is provided by the presence of the condensate, the momentum distribution in the rest of the Brillouin zone is determined by the polaron effect, i.e. by the renormalization of the slave boson wave function due to the strong local scattering from spinons. The latter leads to a strong reduction of the condensate fraction $r_{0}^{2}$, as shown in the inset of Fig.1. The $\omega$-dependence of the dynamic structure factor $N(\mathbf{q}, \omega)$ given by Eq. (3) is shown in Fig. 2 for momenta along the direction $(\pi, 0)$. Results for some selected momenta are presented in Fig.3 together with the exact diagonalization data [1]. We note that the overall energy scale for $N(\mathbf{q}, \omega)$ at large momenta is insensitive to the ratio $J / t$, whereas the value of the spinon bond-order parameter $\chi$, which enters in (3) via the free bosonic dispersion $\omega_{\mathbf{q}}$, is important. In the $N=\infty$ limit $\chi_{\infty} \simeq 2 / \pi^{2}$ is given by that of free fermions, while for the original $t-J$ model its value should be larger [15] due to Gutzwiller projection. For our comparison of $N(\mathbf{q}, \omega)$ (Eq. (3)) with the exact results of Ref. [1] we consider $\chi$ as a free parameter chosing $\chi=\frac{3}{2} \chi_{\infty}$.

The calculated density response function of the $t-J$ model has quite rich structure showing pronounced fea-

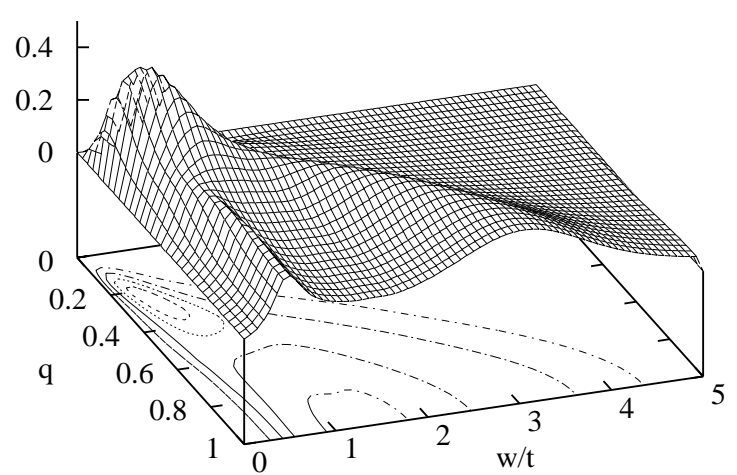

FIG. 2. Density response function $N(\mathbf{q}, \omega)$ for $J / t=0.4$ and $\delta=0.25$ along the $(\pi, 0)$ direction.

tures on different energy scales. The main spectral weight of the excitations at large momenta is located in an energy region of order of several $t$. This high energy peak is very broad and incoherent as a result of the strong coupling of bosons to low-energy spin excitations. We find that the position of this peak and its shape are rather insensitive to the ratio $J / t \leq 1$ in agreement with conclusions of [1.21. This is simply due to the fact that the high-energy properties of the $t-J$ model are controlled by $t$. The theory predicts also a second peak at lower energy (Figs. 2,3) which is more pronounced in the direction $(\pi, 0)$, while its weight is strongly suppressed for q near $(\pi, \pi)$. The origin of this excitation is due to the formation of a polaron-like band of dressed bosons. The relative weight of this contribution increases with $J$ as a result of the increasing spinon bandwidth. As noted above, the density response for small $q$ is mainly given by the collective excitation with energy $\epsilon_{q}=v_{s} q$. The velocity $v_{s}$ is an increasing function of the hole density $\delta$ as expected. Distinct from the one-dimensional model here the collective density ("holon") excitations always overlap with the spinon particle-hole spectrum leading to a strong damping which is also linear in $q$, i.e. $\gamma_{q}=\alpha \epsilon_{q}$. Numerically, at $\delta=0.25$ and $J=0.4 t$ for instance, we found $v_{s} \simeq 0.4 t$ and $\alpha \simeq 0.5$.

Finally we discuss our results for the static structure factor $N(\mathbf{q})=\delta \cdot \int d \omega N(\mathbf{q}, \omega)$, where the factor $\delta$ appears because $N(\mathbf{q}, \omega)$ denotes the normalized density response. Figure 4 shows how effectively the theory presented accounts for the strong suppression of density fluctuations in the vicinity of the Mott transition. The calculated $N(\mathbf{q})$ is a linear function of $q$ for small momenta as a result of the interaction between bosons, and it saturates at a value $N(\mathbf{q}) \simeq \delta$ for large $q$ as it should. Our result for $N(\mathbf{q})$ is very close to that by Gros and Valenti [8], who used the Gutzwiller projected wave function for constrained fermions. It is worth noticing that our re- 


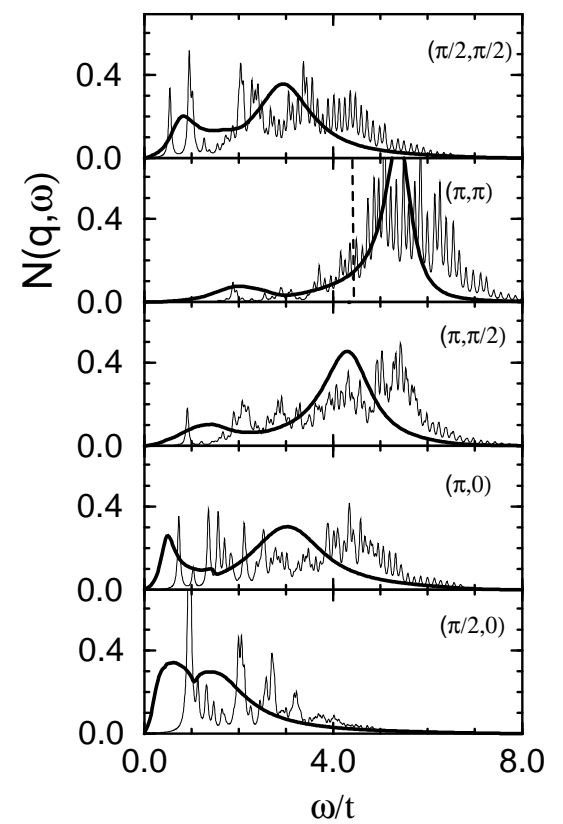

FIG. 3. Comparison of $N(\mathbf{q}, \omega)$ for large momenta with data obtained by exact diagonalization [1] for a $4 \times 4$ periodic cluster with $J / t=0.4$ and $\delta=0.25$. The dashed line in the $(\pi, \pi)$ spectrum indicates the $\delta$-function peak obtained if polaron effects are neglected, i.e. if $\Sigma^{(1 / N)}=0$.

sults fulfil the sum rule $\sum N(\mathbf{q})=\delta(1-\delta)$ for constrained electrons within a few percent accuracy.

In conclusion we have studied the density fluctuation spectrum of the $t$ - $J$ model at moderate doping and zero temperature. Our theory based on the assumption of a Fermi-liquid ground state of this model captures all essential features observed in exact diagonalization studies [1,2,16]. Concerning the high- $T_{c}$ materials we predict in the density response at large momentum transfer a low energy peak with energy of the order of $z \tilde{t}=z(J \chi+t \delta)$ arising from the coherent polaron motion of holes, while the linear collective mode will be changed into a plasmon mode due to the Coulomb interaction. The latter has been already investigated by electron-energy loss techniques [17], however the scale $z \tilde{t}$ required to see the low energy structure in $N(\mathbf{q}, \omega)$ has not yet been reached in these experiments.

We acknowledge useful discussions with P. Prelovšek, T. Tohyama and R. Zeyher, and thank C. Gros for providing their Gutzwiller data. One of us (GKh) would like to thank L. Hedin and the Max-Planck-Institut FKF for the hospitality extended to him during his stay. This work was supported in part by E.U. Grant no ERBCHRX CT94.0438.

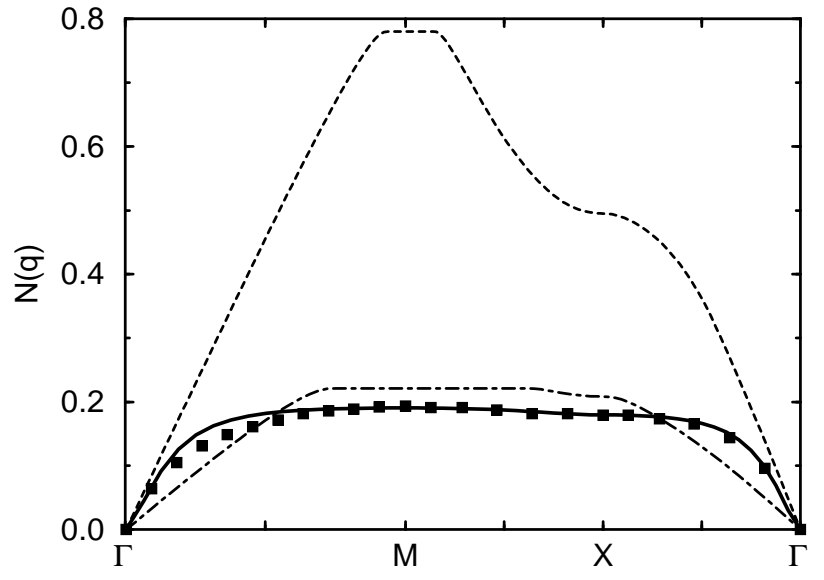

FIG. 4. Comparison of static structure factor $N(\mathbf{q})$ (solid line) calculated for $J=0.4$ with the result for the Gutzwiller wave function of Gros and Valenti [s] (squares) for a hole concentration $\delta=0.213$. For comparison the results for spinless fermions (dash-dotted line) and fermions with spin (dashed line) are shown. The latter comparison shows the large reduction of the density response due to the constraint.

[1] T. Tohyama, P. Horsch, and S. Maekawa, Phys. Rev. Lett. 74980 (1995).

[2] R. Eder, Y.Ohta, and S. Maekawa, Phys. Rev. Lett. 74 5124 (1995).

[3] H.Fukuyama, H. Kohno, B. Normand, and T. Tanamoto, Physica B 213\&214 6 (1995) and references therein.

[4] Z. Wang, Y. Bang, and G. Kotliar, Phys. Rev. Lett. 67, 2733 (1991).

[5] L. Gehlhoff and R. Zeyher, Phys. Rev. 52, 4635 (1995).

[6] D.K.K. Lee, D.H. Kim and P.A. Lee, cond-mat/9601003.

[7] W.O. Putikka, R.L. Glenister, R.R.P. Singh, and H. Tsunetsugu, Phys. Rev. Lett. 73, 170 (1994).

[8] C. Gros and R. Valenti, Phys. Rev. B 50, 11313 (1994).

[9] G. Kotliar and J. Liu, Phys. Rev. B 38, 5142 (1988).

[10] N. Read and D.M. Newns, J. Phys. C 16, 3273 (1983).

[11] E. Arrigoni et al., Physics Reports 241, 291 (1994).

[12] V.N. Popov, Functional Integrals in Quantum Field Theory and Statistical Physics, (D. Reidel, Dordrecht, 1983).

[13] N. Nagaosa and P. Lee, Phys. Rev. Lett. 64, 2450 (1990).

[14] C.L. Kane, P.A. Lee and N. Read, Phys. Rev. B 39, 6880 (1989).

[15] F.C. Zhang, C. Gros, T.M. Rice, and H. Shiba, Supercond. Sci. Technol. 1, 36 (1988); R.B. Laughlin, J. Low Temp. Phys. 90, 443 (1995).

[16] We note that finite-temperature diagonalization studies show no drastic change of $N(\mathbf{q}, \omega)$ up to $T=0.3 t$ (P. Prelovšek, private commun.).

[17] N. Nücker et al., Phys. Rev. B 39, 12379 (1989). 